\title{
Elliptische Randwertprobleme zweiter Ordnung in Gebieten mit einer Fehlstelle
}

\author{
A. Herwig ;
}

Die Lösungen von Randwertproblemen, bei denen das Gebiet durch eine kleine Fehlstelle $\Omega$ e gestört ist, werden nach Potenzen von $\varepsilon$ entwickelt. Dabei soll entweder die Lösung oder ihre Normalableitung auf dem Rand der Fehlstelle verschwinden.

Решения краевых аадач в области с маленкой дырой $\Omega_{c}$ разлагаются в степенной ряд піо $\varepsilon$. Для этого решение или её производная по нормали на границе дыры должно обращаться в нуль.

Solutions of boundary value problems in domains with a small hole $\Omega_{\varepsilon}$ are represented by a power series of $\varepsilon$. For this the solution or its normal derivative should vanish at the boundary of the hole.

0. Seit einiger Zeit werden von verschiedenen Autoren Differentialgleichungsprobleme untersucht, bei denen das betrachtete Gebiet $G$ durch ein Loch in seinem Inneren mit zusätzlichen Forderungen an das Verhalten der Lösung auf dem Lochrand gestört ist. Es wird angenommen, da $\beta$ der Durchmesser der mit $\Omega_{\varepsilon}$ bezeichneten Fehlstelle linear von dem Parameter $\varepsilon$ abhängt und sich beim Grenzübergang $\varepsilon \rightarrow 0$ auf einen einzigen Punkt zusammenzieht. Hierbei handelt es sich um eine singuläre Störung. Während nämlich die Probleme für. Werte aus einem gewissen Parameterbereich korrekt gestellt sind, artet das durch Nullsetzen von $\varepsilon$ entstehende Problem aus. Die zusätzliche Randbedingung kann in einem isolierten Punkt im allgemeinen nicht erfüllt werden.

Zu den ersten Veröffentlichungen zu dieser Thematik zählen die Arbeiten von A. M. IL'IN. Er. behandelt insbesondere ebene Randwertprobleme für elliptische Differentialoperatoren mit variablen Koeffizienten [2, 3]. Aber auch andere Autoren beschäftigen sich mit ähnlichen Aufgabenstellungen. So geben S. Ozawa [7] sowie V. G. Maz'Ja, S. A. Nazarov und B. A. Plamenevsku [5] asymptotische Entwicklungen für die Eigenwerte des Laplace-Operators im gelochten Gebiet an. In anderen Arbeiten (zum Beispiel $[4,6]$ ) werden Gleichungen in Gebieten mit periodisch verteilten Löchern betrachtet, deren. Anzahl beim Grenzprozeß $\varepsilon \rightarrow 0$ gegen unendlich strebt. Die in [1] von D. GöHDE für kugelförmige Defekte angestellten Betrachtungen werden in diesem Artikel bezüglich der zugrunde gelegten Operatoren, Gebiete und der Raumdimension verallgemeinert. Während es in [1] möglich war, die asymptotischen Entwicklungen explizit anzugeben, ist dies bei den hier betrachteten Problemen nicht mehr durchführbar. Besondere Schwierigkeiten bereiten hierbei die ebenen Aufgabenstellungen, da die Lösungen der äußeren' Probleme im allgemeinen nicht so gewählt werden können, daß sie im Unendlichen verschwinden. Daher sind sie zur Kompensation der durch die Lösungen der ausgearteten Probleme auf dem Lochrand verursachten Fehlerterme zunächst ungeeignet.

Seien $G \subset \mathbf{R}^{n}$ und $\Omega \subset \mathbf{R}^{n}$ zwei einfach zusammenhängende und hinreichend glatt berandete Gebiete, die den Koordinatenursprung enthalten. Dann definieren wir ein 
gestörtes Gebiet $G_{\varepsilon}$ durch die Bèziehung $G_{e}=G \backslash \bar{\Omega}_{\varepsilon}$, wobei $\Omega_{\varepsilon}=\left\{x=\left(x_{1}, \ldots, x_{n}\right)\right.$ $\left.\in \mathbf{R}^{n} \mid x / \varepsilon \in \Omega\right\}$ ist, und betrachten das Randwertproblem

$\left.\begin{array}{ll}G_{\varepsilon}: & -\Delta u+c(x)=f(x) \\ \partial G: & u=g(x) .\end{array}\right\}$

unter einèr der beiden zusätzlichen Randbedingungen

$\partial \Omega_{\varepsilon}: \quad u=0$.

bzw.

$\partial \Omega_{\varepsilon}: \quad \partial u / \partial \mathbf{n}=\mathbf{0}$.

Hierbei bezeichnet $\triangle$ den $n$-dimensionalen Laplace-Operator, $\partial / \partial \mathrm{n}$ die Ableitung in Richtung der äußeren Normalen $\mathfrak{n}, \partial G$ und $\partial \Omega_{\varepsilon}$ den Rand des Gebietes $G$ bzw. $\Omega_{\varepsilon}$. Zusätzlich gelte $\cos (\mathrm{n}, x)>0$ für alle Punkte $x \in \partial \Omega_{\varepsilon}$. Des weiteren wird vorausgesetz.t, $\operatorname{da} B f, g$ und $c$ hinreichend glatt sind und $c(x) \geqq 0$ in $G$ gilt. Die Lösungen der so definierten Störprobleme werden im weiteren nach wachsenden Potenzen des Störparameters $\varepsilon$ entwickelt. Hierzu löst man zunächst das ausgeartete Problem (1) in $G$ und addiert einen geeigneten Korrekturterm, der den Einflu $B$ des Defektes zum Ausdruck bringt und möglichst nur in unmittelbarer Nähe der Fehlstelle einen, wesentlichen Beitrag zur Iösung des Störproblems liefert. Den dadurch auf $\partial G$ entstandenen Fehler kompensiert man durch die Lösung eines im unverletzten Gebiet gestellten Problems usw.

1. Zunächst werde der Fall $n \geqq 3$ betrachtet. Hier gilt

Satz 1: Die Lösung u des Problems (1), (2) besitzt im Falle $n \geqq 3$ die asymptotische Darstellung

$$
u=w_{0}+v_{0}+\varepsilon\left(w_{1}+v_{1}\right)+\ldots+\varepsilon^{k}\left(w_{k}+v_{k}\right)+\varepsilon^{k+1} z,
$$

wobei $z$ eine in der Maximumnorm unabhängig von $\varepsilon$ beschränkte Funktion ist. Die Funktion $w_{0}$ genügt dem ausgearteten Problem

$\left.\begin{array}{ll}G: & -\Delta w_{0}+c(x) w_{0}=f(x) \\ \partial G: & w_{0}=g(x)\end{array}\right\}$

und $v_{0}$ 'dem äußeren Dirichlet-Problem

$\left.\begin{array}{l}\mathbf{R}^{n} \backslash \bar{\Omega}_{\varepsilon}: \Delta v_{0}=0 \\ \partial \Omega_{\varepsilon}: \quad v_{0}=-w_{0}(x)\end{array}\right\}$.

Die Funktionen $w_{i}$ sind Lösungen der Gleichungen

$\left.\begin{array}{ll}G: & -\Delta w_{i}+c(x) w_{i}=-\varepsilon^{-1} c(x) v_{i-1} \\ \partial G: & w_{i}=-\varepsilon^{-1} v_{i-1}\end{array}\right\}$

$(i=1,2, \ldots, k)$, und die Funktionen $v_{i}$ genügen den Gleichungen

$$
\left.\begin{array}{l}
\mathbf{R}^{n} \backslash \bar{\Omega}_{\mathrm{e}}: \quad \Delta v_{i}=0 \\
\partial \Omega_{c}: \quad v_{i}=-w_{\mathrm{i}}(x)
\end{array}\right\}
$$


Es sei bemerkt, daß die rechten Seiten der Probleme $\left(5_{i}\right)$ zunächst nur außerhalb von $\Omega_{e}$ definiert sind. Diese können aber leicht stetig auf $\Omega_{\varepsilon}$ fortgesetzt werden, indem man $v_{i-1}$ $=-w_{i-1}$ in $\Omega_{\varepsilon}$ setzt.

Zum Nachweis der punktweisen Beschränktheit von $z$ löst man die Gleichung (4) nach $z$ auf und setzt das Ergebnis in das Störproblem (1), (2) ein. Hierbei ergibt sich

$\left.\begin{array}{ll}G_{\varepsilon}: & -\Delta z+c(x) z=-\varepsilon^{-1} c(x) v_{k} \\ \partial G: & z=-\varepsilon^{-1} v_{k} \\ \partial \Omega_{\varepsilon}: & z=0 .\end{array}\right\}$

Da $z$ unter. Anwendung der Majorisierungsvariante des Maximumprinzips abgeschätzt werden soll, seien einige Eigenschaften der auf den rechten Seiten in $\left(5_{i}\right)$ und (7) auftretenden Lösungen der äußeren Dirichlet-Probleme $\left(6_{0}\right)$ und $\left(6_{i}\right)$ angegeben. Zunächst kann ein Problem der Gestalt

$\left.\begin{array}{l}\mathbf{R}^{n} \backslash \bar{\Omega}_{\varepsilon}: \quad \Delta v=0 \\ \partial \Omega_{\varepsilon}: \quad v=w(x)\end{array}\right\}$

durch die Transformation $x^{\prime}=x / \varepsilon$ in das Problem

$\left.\begin{array}{l}\mathbf{R}^{n} \backslash \bar{\Omega}: \quad \Delta v^{\prime}=0 \\ \partial \Omega: \quad v^{\prime}=w^{\prime}\left(x^{\prime}\right)=w\left(\varepsilon x^{\prime}\right)\end{array}\right\}$

übergeführt werden. Hierbei hängt das Gebiet nicht mehr von dem Parameter $\varepsilon$ ab. Mit Hilfe der, Kelvin-Spiegelung an der Einheitskugel $\dot{y}_{l}=x_{l}^{\prime} /\left|x^{\prime}\right|^{2}(l=1,2, \ldots, n)$ führt man das äußere Problem (9) in das innere Problem

$\left.\begin{array}{ll}\Omega^{\prime}: & \triangle \bar{v}^{\prime}=0 \\ \partial \Omega^{\prime}: & \bar{v}^{\prime}=|y|^{2-n} w^{\prime}(y)=|y|^{2-n} w\left(\varepsilon y /|y|^{2}\right)\end{array}\right\}$

über. Hierbei ist $\Omega^{\prime}=\left\{y^{\prime}=x^{\prime 2} /\left|x^{\prime}\right|^{2} \mid x^{\prime} \in \mathbf{R}^{n} \backslash \bar{\Omega}\right\}$. Damit ist die durch $v^{\prime}\left(x^{\prime}\right)=\left|x^{\prime}\right|^{2-n}$ $\times v^{\prime}\left(x^{\prime} /\left|x^{\prime}\right|^{2}\right)$ definierte Funktion harmonisch in $\mathbf{R}^{n} \backslash \bar{\Omega}$ und erfuillt wegen $\left|x^{\prime}\right|=|y|^{-1}$ die Randbedingung in (9). Die Lösung von Problem (8) lautet

$$
v(x)=(\varepsilon /|x|)^{n-2} \bar{v}^{\prime}\left(\varepsilon x /|x|^{2}\right),
$$

wobei $\bar{v}^{\prime}$ die durch (10) festgelegte Funktion ist. Sei noch $M=\max \left\{|y|^{2-n}\left|w\left(\varepsilon y /|y|^{2}\right)\right|\right.$ $\left.\mid y \in \partial \Omega^{\prime}\right\}$. Dann folgt aus der Anwendung des Maximumprinzips auf (10) die Abschätzung

$$
|v(x)| \leqq(\varepsilon /|x|)^{n-2} M .
$$

Sei nun $C=\max \{c(x) \mid x \in G\}$. Dann genügt die Funktion $z$ aus der Lösungsdarstellung (4) wegen der. Giiltigkeit von (7) dem Ungleichungssystem

$\left.\begin{array}{ll}G_{\varepsilon}: & |-\Delta z+c(x) z| \leqq\left(\varepsilon^{n-3} /|x|^{n-2}\right) C M \\ \partial G: & |z| \leqq\left(\varepsilon^{n-3} /|x|^{n-2}\right) M \\ \partial \Omega_{\varepsilon}: & z=0\end{array}\right\}$

und kann nach der Majorisierungsvariante des Maximumprinzips durch $Z=A$ $-(C M /(n-1))|x|$ abgeschätzt werden. Hierbei ist $A=1+(C M /(n-1))$ $\times \max \{|x| \mid x \in G\}$. Damit gilt $|z(x)| \leqq Z(x)$ für alle $x$ aus $G_{2}$, und die Beschränktheit der Funktion $z$ im Restglied der asymptotischen Lösungsclarstellung (4) ist nachgewiesen. 
Wir betrachten nun das Störproblem (1), (3), bèi clem das Verschwinden der Normalableitung auf dem Lochrand gefordert wird. Hier gilt

Satz 2: Die asymptotische Lösungsdarstellung zu Problem (1), (3) lautet im Falle $n \geqq 3$

$$
u=w_{0}+\varepsilon\left(w_{1}+v_{1}\right)+\ldots+\varepsilon^{k}\left(w_{k}+v_{k}\right)+\varepsilon^{k+2} z,
$$

wobei $z$ wiederum punktweise beschränkt ist. Die Funktionen $w_{0}$ und $w_{i}$ genügen hierbei den Gleichungen $\left(5_{0}\right)$ bzw. $\left(5_{i}\right)(i=1,2, \ldots, k)$, und die Funktionen $v_{0} b z w . v_{i}$ sind Lösungen der äußeren Neumannschen Probleme

$\left.\begin{array}{l}\mathbf{R}^{n} \backslash \bar{\Omega}_{\varepsilon}: \quad A v_{0}=0 \\ \partial \Omega_{\varepsilon}: \quad \partial v_{0} / \partial \mathfrak{n}=-\partial w_{0} / \partial \mathfrak{n}\end{array}\right\}$.

bzw:

$\left.\begin{array}{l}\mathbf{R}^{n} \backslash \bar{\Omega}_{\varepsilon}: \quad \Delta v_{i}=0 \\ \partial \Omega_{\varepsilon}: \quad . \quad \partial v_{i} / \partial \mathfrak{n}=-\partial w_{i} / \partial \mathfrak{n}\end{array}\right\}$

$(i=1,2, \ldots, k)$ :

Zum Nachweis löst man die Beziehung (13) wiederum nach $z$ auf und setzt das Ergebnis in das Störproblem (1), (3) ein. Hierbei ergibt sich

$\left.\begin{array}{ll}G_{\varepsilon}: & -\Delta z+c(x) z=-\varepsilon^{-2} c(x) v_{k} \\ \partial G: & z=-\varepsilon^{-2} v_{k} \\ \partial \Omega_{\varepsilon}: & \partial z / \partial \mathfrak{n}=0 .\end{array}\right\}$

Es ist wiederum das Verhalten der Funktionen $v_{0}$ und $v_{i}$ aus $\left(14_{0}\right)$ bzw. (14 $\left.{ }_{i}\right)$ zu untersuchen. Hierzu fühíren wir die Probleme der Gestalt

$\left.\begin{array}{l}\mathbf{R}^{n} \backslash \bar{\Omega}_{\varepsilon}: \quad \Delta v=0 \\ \dot{\partial} \Omega_{\varepsilon}: \quad \partial v / \partial \mathfrak{n}=\partial w(x) / \partial \mathfrak{n}\end{array}\right\}$

mit Hilfe der Transformation $x^{\prime}=x / \varepsilon$ in das Problem

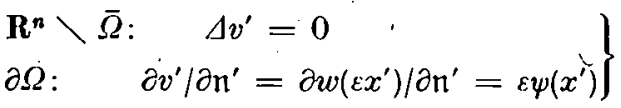

über, wobei $\partial / \partial \mathbf{a}^{\prime}$ die Ableitung in Richtung der äußeren Normalen $n^{\prime}$ an $\partial \Omega$ bezeichnet und $\psi\left(x^{\prime}\right)$ für alle $\dot{x}^{\prime} \in \partial \Omega$ unabhängig von $\varepsilon$ beschränkt ist. Da mit gilt $v(x)=v^{\prime}(x / \varepsilon)$. Nun führt man das äußere Neumannsche Probleni (17) unter Anwendung der KelvinSpiegelung in ein inneres Problem über. Dazu ist es zunächst notwendig, die Transformation des Normalenvektors $\mathfrak{n}^{\prime}$ an $\partial \Omega$ zu beschreiben. Die Fläche $\partial \Omega$ sei durch die implizite Darstellung $\partial \Omega=\left\{x^{\prime} \in \mathbf{R}^{n} \mid F\left(x^{\prime}\right)=0\right\}$ gegeben. Dann gilt für die $s$-te Komponente $\mathrm{n}_{s}{ }^{\prime}\left(x^{\prime}\right)$ von $\mathfrak{n}^{\prime}$ im Punkt $x^{\prime}$ die Beziehung.

$$
\mathfrak{u}_{s}^{\prime}\left(x^{\prime}\right)=\left|\operatorname{gradl}_{x^{\prime}} F\left(x^{\prime}\right)\right|^{-1} \partial F\left(x^{\prime}\right) / \partial x_{s}^{\prime},
$$

wobei $\operatorname{grad}_{x^{\prime}} F\left(x^{\prime}\right)$ den Vektor der partiellen Ableitungen der Funktion $F$ im Punkt $x^{\prime}$ beziiglich der Koordinaten $x_{t}^{\prime}(t=1,2, \ldots, n)$ bezeichnet. Bei der Kelvin-Spiegelung geht die Funktion $F$ in $F^{\prime}$ mit $F^{\prime}(y)=F\left(y /|y|^{2}\right)$ über, und die Komponenten des Normalenvektors $\boldsymbol{y}$ an $\partial \Omega^{\prime}=\left\{y \in \mathbf{R}^{n} \mid F^{\prime}(y)=0\right\}$ lauten

$$
\nu_{s}(y)=\left|\operatorname{grad}_{y} \dot{F}^{\prime}(y)\right|^{-1} \partial F^{\prime}(y) / \partial y_{s} \quad(s=1,2, \ldots, n) .
$$


In den folgenden Formeln ist von 1 bis zur Raumdimension $n$ über doppelt auftretende Indizes zu summieren. Es gilt

$$
\begin{aligned}
\frac{\partial F\left(x^{\prime}\right)}{\partial x_{s}{ }^{\prime}} & =\frac{\partial y_{t}}{\partial x_{s}{ }^{\prime}} \frac{\partial F^{\prime}(y)}{\partial y_{t}}=\left[\frac{\delta_{s t}}{\left|x^{\prime}\right|^{2}}-\frac{2 x_{s}^{\prime} x_{t}^{\prime}}{\left|x^{\prime}\right|^{4}}\right] \frac{\partial F^{\prime}(y)}{\partial y_{s}} \\
& =|y|^{2} \frac{\partial F^{\prime}(y)}{\partial y_{s}}-2 y_{s} y_{t} \frac{\partial F^{\prime}(y)}{\partial y_{t}}=\left|\operatorname{grad}_{\nu} F^{\prime}(y)\right|\left(|y|^{2} \nu_{s}-2 y_{s} y_{t} \nu_{t}\right)
\end{aligned}
$$

$\left(s=1,2, \ldots, n ; \delta_{s t}\right.$ bezeichnet. das Kronecker-Symbol). Nach Quadrieren und Aufsummieren der $n$ Gleichungen (20) erhält man $\left|\operatorname{grad}_{x^{\prime}} F\left(x^{\prime}\right)\right|^{2}=|y|^{4}\left|\operatorname{grad}_{y} F^{\prime}(y)\right|^{2}$. Setzt man die Beziehungen (19) und (20) in (18) ein, so ergibt sich $\mathfrak{n}_{s}^{\prime}\left(x^{\prime}\right)=v_{s}$ $-2\left(y_{s} y_{i} /|y|^{2}\right) v_{\vartheta}$. Wird nun anstelle des zweiten Randwertproblems (17) das dritte Randwertproblem

$\left.\begin{array}{ll}\Omega^{\prime}: & \Delta \bar{v}^{\prime}=0 \\ \partial \Omega^{\prime}: & \partial \bar{v}^{\prime} / \partial v+(n-2) \cos (v, y) /|y| \bar{v}^{\prime}=|y|^{-n} \varepsilon \psi\left(y|y|^{2}\right)\end{array}\right\}$

gelöst, so lautet analog zu oben die Lösung von (17) $v^{\prime}\left(x^{\prime}\right)=\left|x^{\prime}\right|^{2-n} \bar{v}^{\prime}\left(\left.x^{\prime}|| x^{\prime}\right|^{2}\right)$. Die so. definierte Funktion ist wiederum harmonisch in $\mathbf{R}^{n} \backslash \bar{\Omega}$ und genügt wegen

$$
\begin{aligned}
\frac{\partial v^{\prime}\left(x^{\prime}\right)}{\partial \mathfrak{n}^{\prime}} & =\mathfrak{n}_{s}^{\prime} \frac{\partial}{\partial x_{s}^{\prime}}\left[\left|x^{\prime}\right|^{2-n} \bar{v}^{\prime}\left(\frac{x^{\prime}}{\left|x^{\prime}\right|^{2}}\right)\right] \\
& =|y|^{n-2}\left(v_{s}-\frac{2 y_{s} y_{t}}{|y|^{2}} v_{t}\right)\left[(2-n) y_{s} \bar{v}^{\prime}(y)+\left(|y|^{2} \delta_{s l}-2 y_{s} y_{l}\right) \frac{\partial \dot{v}^{\prime}(y)}{\partial y_{l}}\right] \\
& =|y|^{n}\left[(n-2) \frac{\cos (\nu, y)}{|y|} \bar{v}^{\prime}(y)+\frac{\partial \bar{v}^{\prime}(y)}{\partial v}\right]=\varepsilon \psi\left(\frac{y}{|y|^{2}}\right)
\end{aligned}
$$

der Randbedingung in (17). Somit lautet die Lösung von (16) $v(x)=(\varepsilon /|x|)^{n-2} \bar{v}^{\prime}(\varepsilon x \mid$ $\left.|x|^{2}\right)$. Da die Funktion $\psi$ in (17) unabhängig von $\varepsilon$ beschränkt ist, folgt aus dem Maximumprinzip die Existenz einer Konstanten $M$, so daß $\left|v^{\prime}\left(x^{\prime}\right)\right| \leqq \varepsilon M$ für álle $x^{\prime} \in \Omega^{\prime}$. gilt. Folglich genügt $v$ der Beziehung

$$
|v(x)| \leqq \varepsilon(\varepsilon /|x|)^{n-2} M
$$

Damit erfüllt die durch (15) festgelegte Funktion $z$ aus dem Restglied der Lösungsdarstellung (13) die Ungleichungen

$G_{\varepsilon}: \quad|-\Delta z+c(x) z| \leqq\left(\varepsilon^{n-3} /|x|^{n-2}\right) M C$

$\partial G: \quad|z| \leqq\left(\varepsilon^{n-3} /|x|^{n-2}\right) M$

$\partial \Omega_{\varepsilon}: \quad \partial z / \partial \mathrm{n}=0$.

Als Vergleichsfunktion wählen wir hier $Z=1+(A-|x|) \varrho^{n-3} M C /(n-1)$, wobei $\varrho=\max \left\{\varepsilon /|x| \mid x \in \partial \Omega_{e}\right\}$ und $A=\max \{|x| \mid x \in \partial G\}$ ist. Dann gilt

$$
\left.\begin{array}{ll}
-G_{e}: & -\Delta( \pm z-Z)+c(x)( \pm z-Z) \leqq 0 \\
\partial G: & \pm z-Z<0 \\
\partial \Omega_{c}: & \partial( \pm z-Z) / \partial \mathfrak{n}>0 .
\end{array}\right\}
$$

Wegen der Randbedingung auf $\partial \Omega_{\ell}$, die aus der eingangs erwähnten Einschränkung an den Rand des Defektes folgt, wachsen die Funktionen $\pm z-Z$ in Richtung des Gebietsinneren. Deshalb können sie ihr Maximum auf diesem Teil des.Randes von $G_{\varepsilon}$ nicht annehmen. Nach dem Maximumprinzip besitzen sie des weiteren in $G_{\varepsilon}$ 
weder ein negatives Minimum noch ein positives Maximum. Folglich gilt $\pm z(x)$ $\leqq Z(x)$ für alle $x$ aus $G_{\epsilon}$. Damit ist gezeigt, daß das Restglied in der Lösungsdar. stellung (13) zu dem Problem (1), (3) eine Größe der Ordnung $\varepsilon^{k+2}$ ist.

2. Bisher wurde ausschließlich der Fall $n \geqq 3$ betrachtet. Zur asymptotischen Darstellung der Lösung des Störproblems (1), (2) konnte wesentlich ausgenutzt werden, da $B$ die Lösungen der äußeren Probleme $\left(6_{0}\right)$ und $\left(6_{i}\right)$ beim Grenzübergang $|x| / \varepsilon \rightarrow \infty$ gegen Null gehen. In der Ebene bessitzen diese Funktionen eine derartige Eigenschaft nicht. Daher sind zur Konstruktion einer (4). entsprechenden Entivicklung noch einige weitere Betrachtungen anzustellen.. Sei nun $n=2$. Wir betrachten wiederum das äußere Dirichlet-Problem (8), welches durch die Transformation $x^{\prime}=x / \varepsilon$ in (9) und durch $y_{l}=x_{l} /|x|^{2}(l=1,2)$ in (10) übergeht. Die Lösung $\bar{v}^{\prime}$ des Problems besitze in einer kleinen Umgebung des Koordinatenursprunges die Entwicklung $\bar{v}^{\prime}(y)$ $=\bar{v}^{\prime}(0)+|y| R(y)$ mit Restglied erster Ordnung, in der $R(y)$ unabhängig von $|y|$ beschränkt ist. Dann läßt sich die Lösung $v$ des Problems (8) wegen

$$
v(\dot{x})=v^{\prime}\left(\frac{x}{\varepsilon}\right)=\bar{v}^{\prime}\left(\frac{\varepsilon x}{|x|^{2}}\right)=\bar{v}^{\prime}(0)+\frac{\varepsilon}{|x|} R\left(\frac{\varepsilon x}{|x|^{2}}\right)=v(\infty)+\frac{\varepsilon}{|x|} R\left(\frac{\varepsilon x}{|x|^{2}}\right)
$$

für hinreichend große Werte von $|x| / \varepsilon$ als Summe aus einer Konstanten und einem Term, der sich wie $\varepsilon /|x|$ verhält und harmonisch ist, darstellen.

Sei nun $w_{00}$ die Lösung des ausgearteten Problems

$\left.\begin{array}{ll}G: & -\Delta w_{00}+c(x) w_{00}=f(x) \\ \partial G: & w_{00}=g(x)\end{array}\right\}$

und $v^{0}$ die Lösung des äußeren Dirichlet-Problems

$\left.\begin{array}{l}\mathbf{R}^{2} \backslash \bar{\Omega}_{\varepsilon}: \quad \Delta v^{0}=0 \\ \partial \Omega_{\varepsilon}: \quad v^{0}=-w_{00}(x)\end{array}\right\}$

Dann ist $v^{0}$ zur Kompensation von $w_{00}$ auf $\partial \Omega_{\varepsilon}$ zunächst ungeeignet, da $v^{0}$ auf $\partial G$ mit $\varepsilon$ nicht gegen Null geht. Daher kompensiert man unter Verwendung der Darstellung (23) mit der in $G_{\varepsilon}$ harnionischen Funktion $v^{0}(\infty)(\lambda-\ln r+\gamma(x)) /(\lambda-\ln \varepsilon)$ $+\left(v^{0}(x)-v^{0}(\infty)\right)=\mu v_{01}+\varepsilon v_{10}$. Hierbei ist $r=|x|, \quad \mu=(\hat{\lambda}-\ln \varepsilon)^{-1}, \quad v_{10}(x)=$ $=\varepsilon^{-1}\left(v^{0}(x)-v^{0}(\infty)\right), v_{01}(x)=v^{0}(\infty)(\lambda-\ln r+\gamma(x))$ und $\lambda$ eine noch frei wählbare Konstante. $\gamma$ sichert als liösung des Problems

$\left.\begin{array}{lr}\mathbf{R}^{2} \backslash \bar{\Omega}_{\varepsilon}: & \Delta \gamma=0 \\ \partial \Omega_{\varepsilon}: & \gamma=\dot{\ln }(r / \varepsilon),\end{array}\right\}$

da $\beta v^{0}$ und $\mu v_{01}+\varepsilon v_{10}$ auf $\partial \Omega_{\varepsilon}$ übereinstimmien. Damit wird erreicht, da $\beta$ die nullte Korrekturfunktion auf $\partial G$ mit $\varepsilon$ gegen Null geht. Die Lösüngsdarstellung mit Restglied der Ordnung $\varepsilon$ lautet

$$
u(x)=w_{00}(x)+\mu v_{01}(x)+\varepsilon v_{10}^{2}(x)+\mu z .
$$

Ist man an Näherungen höherer Ordnung interessiert, so löst man als nächstes das Problem

$\left.\begin{array}{ll}G: & -\Delta w_{10}+c(x) w_{10}=-c(x) v_{10}(x) \\ \partial G: & w_{10}=-v_{10}(x)\end{array}\right\}$. 
und kompensiert $\varepsilon v_{10}$ auf $\partial G$ durch $\varepsilon w_{10}$. Nun ist der durch $\mu v_{01}$ auf $\partial G$ verursachte Fehler auszugleichen. Dazu löst man das Ersatzproblem

$\left.\begin{array}{ll}G: & -\Delta \bar{w}+c(x) \bar{w}=\dot{c}(x)(\gamma(x)-\ln r) \\ \partial G: & \bar{w}=\gamma(x)-\ln r,\end{array}\right\}$

setzt $\lambda=-\bar{w}(0)$ und kompensiert $\mu v_{01}$ auf $\partial G$ durch die Funktion $\mu w_{01}(\dot{x})=\mu v^{0}(\infty)$ $(\lambda+\bar{w}(x))$. Dürch den so von A. M. IL'rv bereits in [3] eingeführten Parameter $\lambda$ wird gesichert, da $B$ die auftretenden Reihen nach wachsenden Potenzen von $\mu$ nach endlich vielen Gliedern abbrechen. Damit ist $w_{01}$ auf $\partial \Omega_{\varepsilon}$ nämlich eine.Größe der Ordnung $\varepsilon$, und man gelangt zu Restgliedern, deren Ordnung nicht nur eine Potenz von $\mu$, sondern eine Potenz von $\varepsilon$ ist. Die Lösungsdarstellung mit Restglied der.Ordnung $\varepsilon$ lautet somit

$$
u=w_{00}+\varepsilon v_{10}+\mu v_{01}+\varepsilon w_{10}+\mu w_{01}+\varepsilon z .
$$

Im folgenden Schritt sind die Probleme

$\left.\begin{array}{lc}\mathbf{R}^{2} \backslash \bar{\Omega}_{\varepsilon}: \quad & v^{10}=0 \\ \partial \Omega_{\varepsilon}: & v^{10}=-w_{10}\end{array}\right\}$

und

$\left.\begin{array}{lcc}\mathbf{R}^{2} \backslash \bar{\Omega}_{\varepsilon}: & v^{11}=0 \\ \partial \Omega_{\varepsilon}: & v^{11}=-\varepsilon^{-1} w_{01}\end{array}\right\}$

zu lösen. Nun setzt man $v_{20}(x)=\varepsilon^{-i}\left(v^{10}(x)-v^{10}(\infty)\right), v_{11}(x)=\dot{v}^{10}(\infty)(\ln r-\lambda+$ $\gamma(x)), \quad v_{21}(x)=\varepsilon^{-1}\left(v^{11}(x)-v^{11}(\infty)\right), \quad v_{12}(x)=v^{11}(\infty)(\ln r-\lambda+\gamma(x)) \quad$ und $:$ kompensiert $v_{11}$ und $v_{12}$ auf $\partial G$ durch $w_{11}(x)=v^{10}(\infty)(\dot{\lambda}+\bar{w}(x))$ bzw. $w_{1 \dot{2}}(x)=v^{11}(\infty)$ $(\lambda+\bar{w}(x)) ; w_{11}$ und $w_{12}$ sind auf $\partial \Omega_{\epsilon}$ Größen der Ordnung $\varepsilon ; v_{20}$ und $v_{21}$ sind auf $\partial G$ beschränkt. Daher sind diese Terme erst im nächsten Schritt auszugleichen. Es gilt

Satz 3: Die Lösung $u$ des Problems (1), (2) kann für $n=2$ durch

$$
\begin{aligned}
u= & w_{00}+\varepsilon v_{10}+\mu v_{01}+\varepsilon w_{10}+\mu w_{01} \\
& +\varepsilon^{2} v_{20}+\varepsilon^{2} \mu v_{21}+\varepsilon \mu v_{11}+\varepsilon \mu^{2} v_{12}+\varepsilon \mu w_{11}+\varepsilon \mu^{2} w_{12}+\varepsilon^{2} z
\end{aligned}
$$

asymptotisch dargestellt werden. Dabei ist z unabhängig von $\varepsilon$ beschränkt.

Führt man das beschriebene Verfahren fort, so gelàngt man zu asymptotischen Lösungsdarstellungen mit Restgliedern höherer Ordnung in $\varepsilon$.

Die Einführung eines zweiten Parameters neben $\varepsilon$ ist zur Entwicklung der Lösung des ebenen Problems (1), (3) nicht notwendig. Allerdings kanin hier der durch die Lösung $w_{0}$ des ausgearteten Problems auf $\partial \Omega_{c}$ entstandene-Fehler im Gegensatz zum höherdimensionalen Fall nicht durch eine Funktion $v_{0}$, die $\left(\mathbf{1 4}_{\mathbf{0}}\right)$ genügt, kompensiert werden. Das Problem (14 $\left.{ }_{0}\right)$ ist für $n=2$ im allgemeinen unlösbar. Es gilt der

Satz 4: Die Lōsung $u$ des Problems (1), (3) besïtzt für $n=2$ die asymptotische Darslellung

$$
\begin{aligned}
u= & w_{0}+v_{0}+\varepsilon^{2} K_{0} \ln r+\varepsilon\left(w_{1}+v_{1}+\varepsilon^{2} K_{1} \ln r\right) \\
& +\ldots+\varepsilon^{k}\left(w_{k}+v_{k}+\varepsilon^{2} K_{k} \ln r\right)+\left(\varepsilon^{k+2} \ln \varepsilon\right) z,
\end{aligned}
$$


wobei die Funktionen $w_{0}, v_{0}$ und $w_{i}, v_{i}(i=1,2, \ldots, k)$ sowie die Konstanten $K_{0}, K_{i}$ $(i=1,2, \ldots, k)$ den folgenden Beziehungen genügen:

$\left.\begin{array}{ll}G: & -\Delta w_{0}+c(x) w_{0}=f(x) \\ \partial G: & w_{0}=g(x), \\ & K_{0}=-\left(2 \pi \varepsilon^{2}\right)^{-1} \int_{\Omega_{\varepsilon}} \Delta w_{0} d x,\end{array}\right\}$

$\left.\begin{array}{l}\mathbf{R}^{2} \backslash \bar{\Omega}_{\varepsilon}: \quad \Delta v_{0}=0 \\ \partial \Omega_{c}:-\ldots \partial v_{0} / \partial \mathfrak{n}=-\partial\left(w_{0}+\varepsilon^{2} K_{0} \ln r\right) / \partial \mathfrak{n}\end{array}\right\}$

und

$\begin{array}{ll}G: & -\Delta w_{i}+c(x) w_{i}=-\varepsilon^{-1} c(x)\left(v_{i-1}+\varepsilon^{2} K_{i-1} \ln r\right) \\ \partial G: & w_{i}=-\varepsilon^{-1}\left(v_{i-1}+\varepsilon^{2} K_{i-1} \ln r\right)\end{array}$

$$
K_{i}=-\left(2 \pi \varepsilon^{2}\right)^{-1} \int_{\Omega_{e}} \Delta w_{i} d x
$$

$\left.\begin{array}{l}\mathbf{R}^{2} \backslash \bar{\Omega}_{\varepsilon}: \quad \Delta v_{i}=0 \\ \partial \Omega_{\varepsilon}: \quad \partial v_{i} / \partial \mathfrak{n}=-\partial\left(w_{i}+\varepsilon^{2} K_{i} \ln r\right) / \partial \mathfrak{n} .\end{array}\right\}$

Zum Beweis betrachten wir zunächst ein Problem der Gestalt

$\left.\begin{array}{l}\mathbf{R}^{\dot{2}} \backslash \bar{\Omega}_{\varepsilon}:{ }^{\prime} \Delta v=0 \\ \partial \Omega_{\varepsilon}: \quad, \partial v / \partial \mathfrak{n}^{\prime}=-\partial\left(w+\varepsilon^{2} K \ln r\right) / \partial \mathrm{n}, \cdot\end{array}\right\}$

welches genau dann beschränkt lösbar ist; wenn $\int_{\partial \Omega_{\varepsilon}}(\partial v / \partial \mathfrak{n}) d o=0$ gilt. Auus dieser Beziehung berechnen wir $K$. Aus der ersten Greenschen Formel folgt

$$
0=\int_{\partial \Omega_{e}} \frac{\partial w}{\partial \mathfrak{n}} d \sigma_{r}+\varepsilon^{2} K \int_{\partial Q_{e}} \frac{\partial \ln r}{\partial \mathfrak{n}} d \sigma=\int_{\Omega_{\varepsilon}} \Delta \dot{w} d \dot{x}+2 \pi \varepsilon^{2} K .
$$

Es ergibt sich $K=-\left(2 \pi \varepsilon^{2}\right)^{-1} \int_{\Omega_{\varepsilon}} \Delta w d x$, so $\mathrm{da} B$ zunächst die obige Definition der Konstanten $K_{0}$ und $K_{i}(i=1,2, \ldots, k)$ sinnvoll ist. Die Anwendung der Transformation $x^{\prime}=x / \varepsilon$ und der Kelvin-Spiegelung auf (27). liefert das innere Neumannsche Problem.

$\left.\begin{array}{ll}\Omega^{\prime}: & \Delta \bar{v}^{\prime},=0 \\ \partial \Omega^{\prime}: . & \partial \bar{v}^{\prime} / \partial \nu=\varepsilon|y|^{-2} \psi\left(y /|y|^{2}\right) .\end{array}\right\}$

Hierbei ist $\psi$ durch die Beziehung $\psi(x)=-\varepsilon^{-1} \partial w\left(\varepsilon x^{\prime}\right) / \partial \mathfrak{n}^{\prime}-\varepsilon K \partial \ln r^{\prime} / \partial \mathfrak{n}^{\prime}, r^{\prime}=\left|x^{\prime}\right|$, definiert und unabhängig von $\varepsilon$ beschränkt. Die Lösung $\bar{v}^{\prime}$ von (28) ist lediglich bis auf einen konstanten Summanden eindeutig bestimmt. Diesen wählen wir so, da $B$ $\bar{v}^{\prime}(0)=0$ gilt. Weil $\partial \bar{v}^{\prime}(y) / \partial v$ auf $\partial \Omega^{\prime}$ eine Größe der Ordnung $\varepsilon$ ist, ergibt sich $\left|\bar{v}^{\prime}(y)\right|$ $\leqq \varepsilon M$ mit einer gewissen Konstanten $M$ für alle $y \in \Omega^{\prime}$. Nun kann man aus der Lipschitz-Stetigkeit von $\bar{v}^{\prime}$ die Ungleichung $\left|v^{\prime}(y)\right| \leqq \varepsilon \dot{M}^{\prime}|y|$ für $y \in \Omega^{\prime}$ folgern. Entsprechend Formel (11) erhält man die Lösung $v$ von (27) durch den Übergang zu den ursprünglichen Koordinaten: $v(x)=\vec{v}^{\prime}\left(\varepsilon x /|x|^{2}\right)$. Damit genügt $v$ der Abschätzung $|v(x)| \leqq \varepsilon^{2} M^{\prime}|| x \mid$ außerhalb von $\Omega_{\varepsilon}$, und die Funktionen $v_{0}, v_{i}(i=1,2, \ldots, k)$ sind als Lösungen von $\left(26_{0}\right),\left(26_{i}\right)$ auf $\partial \Omega_{\varepsilon}$ Größen der Ordnung $\varepsilon$. Deshalb können die rechten Seiten von $\left(2 \tilde{5}_{i}\right)$ stetig so auf $\Omega_{\boldsymbol{t}}$ fortgesetzt werden; daß diese Eigenschaft 
erhalten bleibt. Zum Nachweis der Beschränktheit von $z$ löst man wiederum die Entwicklung (24) nach $z$ auf und setzt in das Ausgangsproblem (1), (3) ein. Hierbei ergibt sich

$\left.\begin{array}{l}G \backslash \bar{\Omega}_{\varepsilon}: \quad-\Delta z+c(x) z=-\left(\varepsilon^{2} \ln \varepsilon\right)^{-1} c(x)\left(v_{k}+\varepsilon^{2} K_{k} \ln r\right) \\ \partial G: \quad z=-\left(\varepsilon^{2} \ln \varepsilon\right)^{-1}\left(v_{k}+\varepsilon^{2} K_{k} \ln r\right) \\ \partial \Omega_{\varepsilon}: \quad . \quad \partial z / \partial \mathrm{n}=0 . \quad \cdots\end{array}\right\}$

Analog zum oben behandelten höherdimensionalen Fall zeigt man hier unter Anwendung des Maximumprinzips, da $B$ z unabhängig von $\varepsilon$ beschränkt ist.

\section{LITERATUR}

[1] GöнDE, D.: Singuläre Störungen von Randwertproblemen durch ein kleines Loch im Gebiet. Z. Anal. Anw. 4 (1985), 467-477.

[2] Ильин, А. М.: Краевая залача эллиитического уравнения второго порядка в области с узной шелью. 1. Двумерный случай. Мат. сб. 99 (1976), 514-537.

[3] Ильин, А. М.: Краевая задача әллиптического уравнения второго порядка в области с узкой щелью. 2. Область с малым отверстием. Мат. сб. 103 (1977), 265-284.

[4] Иосифьян, Г. А., ОлеЙнин, О. А., и А. С. ШАмаев: О СХодомости әнергии, тензоров напряжений и частот собственных колебаний в задачах усреднения, возникающих в теории упругости. Докл. Акад. Наук СССР 274 (1984), 1329-1333.

[5] МАзья, В. Г., НАзАРов, С. А., и В. А. ПлАмЕнЕВСКИЙ: АСимптотические разложения собственных чисел краевых задач для операторов Лапласа в областях с малыми отверстиями. Изв. Акад. Наук СССР, Сер. Мат., 48 (1984), 347-371.

[6] Nouetzeno, G.: Problèmes d'écrans perforés pour l'équation de Laplace. Rairò Modèl.

: Math. Anal. Numér. 19 (1985), 33-63.

[7] Ozawa, S.: An asymptotic formula for the eigenvalues of the laplacian in a domain with a small hole. Proc. Japan. Acad. Tokyo A 58 (1982), 5-8.

Manuskripteingang: 05. 10. 1987; in revidierter Fassung 28. 03. 1988

VERFASSER :

Dr. AJjred Herito

Heinrich-Heine-Str. 59

DDR-9550 Zwickau 